\title{
Diffeomorphic image matching with left-invariant metrics
}

\author{
Tanya Schmah and Laurent Risser and François-Xavier Vialard*
}

\begin{abstract}
The geometric approach to diffeomorphic image registration known as large deformation by diffeomorphic metric mapping (LDDMM) is based on a left action of diffeomorphisms on images, and a right-invariant metric on a diffeomorphism group, usually defined using a reproducing kernel. We explore the use of left-invariant metrics on diffeomorphism groups, based on reproducing kernels defined in the body coordinates of a source image. This perspective, which we call Left-LDM, allows us to consider non-isotropic spatially-varying kernels, which can be interpreted as describing variable deformability of the source image. We also show a simple relationship between LDDMM and the new approach, implying that spatially-varying kernels are interpretable in the same way in LDDMM. We conclude with a discussion of a class of kernels that enforce a soft mirror-symmetry constraint, which we validate in numerical experiments on a model of a lesioned brain.
\end{abstract}

\section{Introduction}

The geometric point of view on diffeomorphic image matching was pioneered by $[10,31]$, and has been developed significantly in the last ten years $[4,32,21,20$, $9,5,14]$. In its many practical applications to medical imaging, including compu-

\footnotetext{
Tanya Schmah

Rotman Research Institute, Baycrest, and University of Toronto,

e-mail: tschmah@research.baycrest.org

Laurent Risser

CNRS - Institut de Mathématiques de Toulouse, e-mail: 1risser@math.univ-toulouse.fr

François-Xavier Vialard

Université Paris-Dauphine, e-mail: vialard@ ceremade.dauphine.fr

* All authors contributed equally to this work.
} 
tational anatomy [23], the approach is known as the Large Deformation Diffeomorphic Metric Mapping framework (LDDMM). A good geometric overview may be found in [5]. Two key elements of this framework are: a right-invariant Riemannian metric on a group of diffeomorphisms; and the left action of this group on images $I: \Omega \rightarrow \mathbb{R}^{d}$ defined by $\phi \cdot I:=I \circ \phi^{-1}$. Combining these two elements gives an induced Riemannian metric on the group orbit of a given image $I$.

In image registration in general, the inexact matching problem is, given two images $I$ and $J$, to find a transformation $\phi$ that minimises the sum of some measure of the size of $\phi$ and some measure of image dissimilarity (or error) $E(\phi \cdot I, J)$, such as $\|\phi \cdot I-J\|_{L^{2}}^{2}$. In LDDMM, we seek a path of diffeomorphisms $\phi(t)$ starting at $I d$, with the size of the final diffeomorphism $\phi(1)$ given by the length of the path $\phi$ defined by the right-invariant Riemannian metric associated with some norm $\|\cdot\|_{V}$ on a Hilbert space $V$ of smooth vector fields. Thus the fundamental optimisation problem in LDDMM is to minimise

$$
\mathscr{J}(\phi)=\frac{1}{2} \int_{0}^{1}\|v(t)\|_{V}^{2} \mathrm{~d} t+E(\phi(1) \cdot I, J)
$$

for a path $\phi$ with $\phi(0)=I d$, under the constraint

$$
\partial_{t} \phi(t)=v(t) \circ \phi(t)
$$

which defines $v(t)$ as the spatial (Eulerian) velocity of $\phi(t)$. Note that all minimisers of this functional are geodesics, since they must minimise the first term of (1) for a given $\phi(1)$.

The minimisation problem (1) is well-posed provided that the norm on $V$ is sufficiently strong in terms of smoothness (see [36], Theorem 11.2). The Hilbert space $V$ is usually defined via its reproducing kernel:

$$
\|v\|_{K}^{2}=\langle p, K \star p\rangle_{L^{2}}, \text { where } v=K \star p .
$$

A Gaussian kernel is often chosen for computational convenience, or a mixture of Gaussian kernels as in [26, 27].

We note that LDDMM is not the only diffeomorphism-based approach to image matching. There is another family of successful methods, based on exponentiating stationary vector fields $[2,33,3]$. However, unlike these methods, LDDMM is able to draw on concepts in geometry and mechanics such as geodesic distance and momentum, which have been central both to theoretical developments and to recent efficient numerical algorithms [34, 7].

Though not required by the theory, in practice the kernel used in diffeomorphic methods (LDDMM and the other methods cited above) has always been chosen to be translationally-invariant and isotropic. In LDDMM, spatially-varying or nonisotropic ("direction-dependent") kernels have no obvious interpretation, because the norm is defined in Eulerian coordinates, so that as $t$ varies during the deformation, a fixed point in the source image moves through space, and conversely, a fixed point in space will correspond to different points on the source image. Similarly, 
the directions in a direction-dependent kernel are defined with respect to Eulerian coordinates, not the coordinates of the moving source image. Nonetheless, spatiallyvarying kernels are potentially of great interest in medical applications, if they can be made to represent spatially-variable (or non-isotropic) deformability of tissue. This is indeed already done in [25] to model sliding conditions between the lungs and the ribs. In general it is well-known that a good choice of kernel (the "regulariser") is essential for optimising registration performance, so that taking into account any spatial variability of the tissue deformability in the kernel will improve the registration.

With this motivation, we propose a new registration framework, which will support natural interpretations of spatially-varying metrics. Left-Invariant LDDMM ("Left-LDM") is analogous to LDDMM but based on a left-invariant metric, i.e. based on a norm in the body (Lagrangian) coordinates of the source image. This means that instead of the norm in (1) being applied to the spatial (Eulerian) velocity defined by (2), it is applied to the convective velocity defined by

$$
\partial_{t} \phi(t)=d \phi(t) \cdot v(t),
$$

where $d \phi(t)$ is the spatial derivative of $\phi(t)$. To emphasize the relationship between the two frameworks, we will refer to LDDMM from now on as "Right-LDM", consistent with the use of the shortened acronym LDM in [14]. The matching problem in Left-LDM is to minimize the same functional as in Right-LDM (1) but under the "new" constraint (4). Note that the convective velocity of a given $\phi(t)$ is the pullback of the spatial velocity by $\phi_{t}$, i.e. it is just the spatial velocity expressed in body (Lagrangian) coordinates.

Subject to some analytical subtleties explored in Section 2, the solutions $\phi(t)$ are left-geodesics in a diffeomorphism group. The description of left-geodesic flow in terms of the convective velocity is an example of a convective representation of a continuum theory. Convective representations were introduced in [18] for ideal fluid flow, and [29] for elasticity, and the subject has been further developed in [15]. The relationship between left- and right- geodesic flows on a diffeomorphism group was explored earlier in [16].

In the Left-LDM framework, a spatially-varying or non-isotropic kernel makes sense, because it is defined in Lagrangian coordinates, so it can model variable deformability of different parts of the source image. (The norm is carried along by push-forwards with the moving source image.) This opens up possibilities for application-specific regularisation, either hand-tuned or learnt from data.

\section{Analytical setting}

We consider the convective velocity constraint, formula (4), and the conditions on $v(t)$ such that it can be integrated to produce the diffeomorphism $\phi(t)$. Such an evolution equation is a partial differential equation that belongs to the class of linear 
symmetric hyperbolic systems [12]. The usual method for solving such equations consists in using the method of characteristics, which amounts to solve an equation of the type (2) on the inverse of the flow. The equation of characteristics, being equivalent to formula (2), is an ordinary differential equation and can be integrated provided sufficient smoothness assumptions on the spatial velocity. For the spatial velocity constraint, a satisfactory answer has been given in [36, Theorems 8.7 and 8.14]: The flow of a time dependent vector field in $L^{2}([0,1], V)$ is well-defined if there exists a constant $C>0$ such that for every $v \in V$

$$
\|v\|_{1, \infty} \leq C\|v\|_{V}
$$

where $\|v\|_{1, \infty}$ is the Banach norm in $W^{1, \infty}\left(\Omega, \mathbb{R}^{d}\right)$. Under this hypothesis, the variational problem (1) is well-posed and the set $G_{R}$, defined by ${ }^{2}$

$$
G_{R}:=\left\{\phi(1) \mid \partial_{t} \phi(t)=v(t) \circ \phi(t) \text { and } v \in L^{2}([0,1], V), \phi(0)=I d\right\},
$$

is a group. A similar approach in [11] proves that the flow of $v \in C\left([0,1], H^{s}\right)$ defines an $H^{s}$ diffeomorphisms for $s>d / 2+2$. From a variational point of view the former approach is better suited for solving Problem (1). In particular, working with the space $L^{2}([0,1], V)$ is crucial for proving the existence of a minimizer and therefore we cannot reduce our work to a smooth setting. This is our main motivation for developing the following analytical study. Let us then define the following set,

$$
G_{L}:=\left\{\phi(1) \mid \partial_{t} \phi(t)=d \phi(t) \cdot v(t) \text { and } v \in L^{2}([0,1], V), \phi(0)=I d\right\} .
$$

Integrating equation (4) is straightforward in a smooth setting. Indeed, this equation is equivalent to

$$
\partial_{t} \phi^{-1}(t)=-v(t) \circ \phi^{-1}(t) .
$$

Unfortunately, working with $L^{2}([0,1], V)$ vector fields, Equation (8) has to be proven true in that context. An example of this issue is the following: with a fixed regularity, for instance the group Diff $f^{s}$ of $H^{s}$ diffeomorphisms, the inversion map is only continuous and not differentiable. This comes from the fact that the inversion map Inv : Diffs $\rightarrow$ Diffs presents a loss of regularity when being differentiated:

$$
D \operatorname{Inv}(\phi)(v)=-d \phi^{-1}\left(v \circ \phi^{-1}\right) .
$$

The rest of the section will be devoted to show that equation (4) can be solved via the method of characteristics. Our strategy consists in proving that Equation (8) holds under very weak conditions so that integration of the convective velocity equation (4) reduces to the integration of Equation (8).

In what follows, we consider $\Omega$ a closed, bounded domain and $V$ a Hilbert space of vector fields $u$ such that both $u$ and $d u$ vanish on its boundary, and we suppose that $V$ is embedded in $C^{1}\left(\Omega, \mathbb{R}^{d}\right)$, i.e. there exists a constant $C>0$ such that (5) applies for all $u$. Let us begin with the following lemma:

\footnotetext{
${ }^{2}$ In the corresponding definition in [36], $v$ need only be absolutely integrable in time.
} 
Lemma 1. Let $B:=C^{0}\left([0,1], C_{\infty}^{1}\left(\Omega, \mathbb{R}^{d}\right)\right) \cap H^{1}\left([0,1], L^{2}\left(\Omega, \mathbb{R}^{d}\right)\right)$. Let $\phi \in B$, and denote by $\phi^{-1}$ the map $t \mapsto \phi_{t}^{-1}$. If $\phi_{t}$ is a diffeomorphism onto $\Omega$ for all $t \in[0,1]$, then $\phi^{-1}$ lies in $B$.

Remark 1. The subscript $\infty$ denotes the use of the sup norm.

Proof. The standard Inverse Function Theorem implies that $\phi_{t}^{-1}$ is $C^{1}$ for all $t \in$ $[0,1]$. The continuity of $\phi$ implies the continuity of the map $(t, x) \mapsto \phi_{t}(x)$, which by a lesser-known version of the Implicit Function Theorem (see [22]) implies the continuity of $t \mapsto \phi_{t}^{-1}(x)$ for every $x$. Therefore, by compactness of $\Omega$ we have $\phi^{-1} \in C^{0}\left([0,1], C_{\infty}^{1}\left(\Omega, \mathbb{R}^{d}\right)\right.$.

Let us first suppose that $\phi \in C:=C^{0}\left([0,1], C_{\infty}^{1}\left(\Omega, \mathbb{R}^{d}\right) \cap C^{1}\left([0,1], C^{0}\left(\Omega, \mathbb{R}^{d}\right)\right)\right.$, and that (as before) $\phi_{t}$ is a diffeomorphism onto $\Omega$ for all $t \in[0,1]$. Then for all $x \in \Omega$ one has by simple differentiation

$$
\partial_{t} \phi_{t}^{-1}(x)=-\left[d \phi_{t}\right]_{\phi_{t}^{-1}(x)}\left(\partial_{t} \phi_{t}\left(\phi_{t}^{-1}(x)\right)\right) .
$$

We aim at proving that $\partial_{t} \phi_{t}^{-1}$ belongs to $L^{2}\left([0,1], L^{2}\left(\Omega, \mathbb{R}^{d}\right)\right)$ : The first term $\left[d \phi_{t}\right]_{\phi_{t}^{-1}(x)}$ is continuous (on $\Omega$ ) and its sup norm is uniformly bounded for $t \in[0,1]$ since $C_{\infty}^{0}\left([0,1], C_{\infty}^{1}\left(\Omega, \mathbb{R}^{d}\right)\right)$. By assumption, $\partial_{t} \phi_{t} \in L^{2}\left(\Omega, \mathbb{R}^{d}\right)$ and the right composition with a $C^{1}$ diffeomorphism is a bounded linear operator on $L^{2}\left(\Omega, \mathbb{R}^{d}\right)$ (by a standard change of variable). It follows easily that $\partial_{t} \phi_{t}^{-1} \in L^{2}\left([0,1], L^{2}\left(\Omega, \mathbb{R}^{d}\right)\right)$ and $\phi^{-1} \in C$.

We will prove a similar result for any $\phi \in B$ : By density of $C$ in $B$, we consider a sequence $\phi_{n} \in C$ converging to $\phi \in B$. In particular, we have

$$
\phi_{n, T}^{-1}(x)=\int_{0}^{T}-\left[d \phi_{n, t}\right]_{\phi_{n, t}^{-1}(x)}\left(\partial_{t} \phi_{n, t}\left(\phi_{n, t}^{-1}(x)\right)\right) \mathrm{d} t .
$$

First, the left-hand side strongly converges in $C_{\infty}^{1}\left(\Omega, \mathbb{R}^{d}\right)$ (by the inverse function theorem) and thus in $L^{2}\left(\Omega, \mathbb{R}^{d}\right)$ to $\phi_{T}^{-1}$.

Second, the right-hand side weakly converges in $L^{2}\left(\Omega, \mathbb{R}^{d}\right)$ to

$$
\int_{0}^{T}-\left[d \phi_{t}\right]_{\phi_{t}^{-1}(x)}\left(\partial_{t} \phi_{t}\left(\phi_{t}^{-1}(x)\right)\right) \mathrm{d} t .
$$

Indeed, let us consider $f \in C^{\infty}\left(\Omega, \mathbb{R}^{d}\right)$ and calculate the $L^{2}$ scalar product

$$
\begin{array}{r}
\left\langle f, \int_{0}^{T}-\left[d \phi_{n, t}\right]_{\phi_{n, t}^{-1}(\cdot)}\left(\partial_{t} \phi_{n, t}\left(\phi_{n, t}^{-1}(\cdot)\right)\right) \mathrm{d} t\right\rangle=\int_{0}^{T}-\left\langle\left[d \phi_{n, t}\right]_{\phi_{n, t}^{-1}(\cdot)}^{*}(f), \partial_{t} \phi_{n, t}\left(\phi_{n, t}^{-1}(\cdot)\right)\right\rangle \mathrm{d} t \\
=\int_{0}^{T}-\left\langle\left[d \phi_{n, t}\right]^{-1 *}\left(f \circ \phi_{n, t}\right), \partial_{t} \phi_{n, t}(\cdot) \mathrm{Jac}\left(\phi_{n, t}\right)\right\rangle \mathrm{d} t
\end{array}
$$

Since $f$ is smooth and $\Omega$ compact, $f$ is uniformly Lipschitz and thus $\left[d \phi_{n, t}\right]^{-1 *}(f \circ$ $\left.\phi_{n, t}\right)$ converges for the sup norm to $\left[d \phi_{t}\right]^{-1 *}\left(f \circ \phi_{t}\right)$. The same convergence holds for $\operatorname{Jac}\left(\phi_{n, t}\right)$ by assumption. This proves the weak convergence on smooth functions, 
which implies the weak convergence in $L^{2}$ (see [35]). Strong and weak limits are equal so that $\left[d \phi_{t}\right]_{\phi_{t}^{-1}(\cdot)}\left(\partial_{t} \phi_{t}\left(\phi_{t}^{-1}(\cdot)\right)\right) \in L^{2}\left([0,1], L^{2}\left(\Omega, \mathbb{R}^{d}\right)\right)$ is the (time) derivative of $\phi_{t}^{-1}$ and the conclusion ensues.

Remark 2. In fact, we could have proven the following stronger result: the inversion map is continuous on an affine subspace $\tilde{B}$ of $B$ defined by $\tilde{B}=\left\{\phi \in B \mid \phi_{t} \in\right.$ Diff $\}$ endowed with the Banach norm $\sup \left(\|\phi\|_{H^{1}},\|\phi\|_{\infty},\left\|\phi^{-1}\right\|_{\infty}\right)$. However, the proof would be a little more involved and the result is not needed in what follows.

Proposition 1. Solutions in B of (4) exist, are unique and are characterized by being solutions of

$$
\partial_{t} \phi^{-1}(t)=-v(t) \circ \phi^{-1}(t) .
$$

Proof. The initial condition is $\phi_{0}=I d$ together with the assumption $\phi \in B$ imply the existence of a positive real number $T>0$ such that $\phi_{t}$ is a diffeomorphism for $t \in[0, T]$. On this interval, the previous lemma gives that $\phi^{-1} \in B$ and $\partial_{t} \phi_{t}=-\left[d \phi_{t}\right]_{\phi_{t}^{-1}(\cdot)}\left(\partial_{t} \phi_{t}\left(\phi_{t}^{-1}(\cdot)\right)\right)$. Since $\partial_{t} \phi_{t}=d \phi(t) \cdot v(t)$, we obtain $\partial_{t} \phi_{t}^{-1}=$ $-v(t) \circ \phi^{-1}(t)$. Using the result [36, Theorem 8.7] on flow integration, we obtain the existence and uniqueness of $\phi^{-1} \in B$ satisfying (13). This implies also existence and uniqueness of solutions in $B$ of (4) on $[0, T]$. The extension for all time $t \in[0,1]$ is straightforward by considering $I=\sup \left\{T>0 \mid \forall t<T, \phi_{t} \in\right.$ Diff $\}$. By construction, $I$ is open and the argument above shows that $I$ is non-empty. Last, $I$ is closed since the flow of $-v(t)$ is a diffeomorphism for all time $t \in[0,1]$ and therefore $I=[0,1]$.

Remark 3. The definition of the space $B$ could have been a little more general using $W^{1,1}\left(\Omega, \mathbb{R}^{d}\right)$ instead of $H^{1}\left(\Omega, \mathbb{R}^{d}\right)$. However, it was not necessary regarding the existence of minimizers of functional (1) under convective velocity constraint.

In light of this result, we modify the definitions of $G_{L}$ and $G_{R}$ to require that $\phi \in B$ :

$$
\begin{aligned}
& G_{L}:=\left\{\phi(1) \in B \mid \partial_{t} \phi(t)=d \phi(t) \cdot v(t) \text { and } v \in L^{2}([0,1], V)\right\}, \\
& G_{R}=\left\{\phi(1) \in B \mid \partial_{t} \phi(t)=u(t) \circ \phi^{-1}(t) \text { and } u \in L^{2}([0,1], V)\right\} .
\end{aligned}
$$

Since $G_{R}$ is closed under inversion, Proposition 1 implies $G_{L}=G_{R}$. Note that the sets of paths $\phi(t)$ in the definitions of $G_{L}$ and $G_{R}$ do not coincide in general. Indeed, these sets of paths correspond to each other by the inverse map, and this inversion shows a loss of regularity for instance on Diff $f^{\prime}$. In the rest of the paper, we will use the notation $G_{V}$ to denote the group $G_{L}=G_{R}$, and by abuse of notation, $G_{L}$ and $G_{R}$ will denote the set of paths generated under the constraint (4) (and respectively (2)) by elements of $L^{2}([0,1], V)$.

The structure of $G_{V}$ is not well-known. In the case of Gaussian kernels, $G_{V}$ is probably included in an ILH-Lie group in the sense of Omori [24]. In general, it is not known whether $G_{V}$ admits a differentiable structure. Nonetheless, the group carries natural left- and right- invariant metrics, as defined in the next section, and isometries should be understood as being between metric spaces. In the case of 
Sobolev spaces, the right-invariant metric is a smooth Riemannian metric, whereas the left-invariant metric is probably not.

Finally, we can now benefit from the existence of minimizers for the functional (1) in the LDDMM framework:

Theorem 1. If $V$ satisfies assumption (5) and $E$ is continuous w.r.t. uniform convergence of $\phi$ on every compact set in $\Omega$, then there exists a minimizer in $G_{V}$ of the functional (1) under the convective velocity constraint (4).

Proof. This follows from [36, Theorem 11.2].

Note that the theorem applies for the usual sum of squared differences similarity measure:

$$
E(\phi)=\left\|I \circ \phi(1)^{-1}-J\right\|_{L^{2}}^{2} .
$$

\section{Left- and right- invariant metrics on diffeomorphism groups}

Proposition (1) proved that the convective velocity constraint (4) is equivalent to

$$
\partial_{t} \phi^{-1}(t)=-v(t) \circ \phi^{-1}(t),
$$

in a general setting.This equation is simply the spatial velocity constraint (2) for $\phi^{-1}$, except with a minus sign. In other words, if the spatial and convective velocities of any path $\phi(t)$ are denoted by $v_{R}^{\phi}$ and $v_{L}^{\phi}$, respectively, then

$$
v_{R}^{\phi^{-1}}=-v_{L}^{\phi} .
$$

As a consequence of this simple fact (well-known in a smooth setting), there are close relationships between Left-LDM and Right-LDM.

On $G_{V}$ a left-invariant metric $d_{L}$ can be defined by

$$
d_{L}(\phi, I d)=\inf \left\{\sqrt{\int_{0}^{1}\left\|v_{L}^{\phi}(t)\right\|_{V}^{2} d t}: \phi(0)=I d \text { and } \phi(1)=\phi\right\} .
$$

A right-invariant metric $d_{R}$ can be defined in the same way but using the spatial velocity $v_{R}^{\phi}$ instead of the convective velocity $v_{L}^{\phi}$. It follows from (15) that

$$
d_{L}(\phi, I d)=d_{R}\left(\phi^{-1}, I d\right) .
$$

As shown in [31], the distance $d_{R}$ is well-defined and makes $G_{V}$ a complete metric space. From (17), it follows that the same is true of $d_{L}$. Between any two diffeomorphisms in $G_{V}$, there exists a path minimising the distance $d_{L}$ (resp. $d_{R}$ ), and such minimising paths will be called left- (resp. right-) geodesics. Note that we have defined geodesics without reference to a Riemannian metric, since we do not know whether $G_{V}$ even has a smooth structure, as discussed earlier. 
The following proposition summarises some elementary properties of these distance metrics, all straightforward consequences of (15) and the definitions.

Proposition 2. 1. The inverse mapping is an isometry:

$$
\begin{aligned}
\left(G_{V}, d_{L}\right) & \rightarrow\left(G_{V}, d_{R}\right) \\
\phi & \rightarrow \phi^{-1}
\end{aligned}
$$

2. $\phi$ is a left-geodesic if and only if $\phi^{-1}$ is a right-geodesic.

3. Left translation is an isometry of $\left(G_{V}, d_{L}\right)$, and right translation is an isometry of $\left(G_{V}, d_{L}\right)$.

4. The left translation of a left-geodesic is a left-geodesic (and similarly for rightgeodesics).

Remark 4. In the context of fluid dynamics, $\phi$ is the usual Lagrangian map, and $\phi^{-1}$ is the "back-to-labels" map. Observation (2) in the above proposition has been exploited before in this context [16].

We now show two correspondences between Left- and Right- LDM.

Lemma 2. Let $\phi(t)$ be a path of diffeomorphisms with spatial velocity $v_{R}^{\phi}(t)$, defined by (2). Define $\psi: t \rightarrow \phi(1) \phi^{-1}(1-t)$, and let $v_{L}^{\psi}(t)$ be its convective velocity, defined by (4). Then $v_{L}^{\psi}(t)=v_{R}^{\phi}(1-t)$.

Proof. From (2) we have, by direct calculation:

$$
\partial_{t} \phi^{-1}(t)=-d \phi^{-1}(t) \cdot v_{R}^{\phi}(t)
$$

so that

$$
\partial_{t} \phi^{-1}(1-t)=d \phi^{-1}(1-t) \cdot v_{R}^{\phi}(1-t)
$$

and therefore,

$$
\partial_{t} \psi(t)=d \psi(t) \cdot v_{R}^{\phi}(1-t) .
$$

Thus $v_{R}^{\phi}(1-t)$ satisfies the relation (4) that defines $v_{L}^{\psi}(t)$.

The following proposition is a direct consequence of the previous lemma. It concerns a generalisation of the matching functional (1), in which the squared path length in the first term is replaced by the integral of a general Lagrangian $l(v(t))$, and the image dissimilarity term $E(\phi(1) \cdot I, J)$ is replaced by a general real-valued function $H(\phi(1))$.

Proposition 3. Let $V$ and $G=G_{L}=G_{R}$ be as defined above. Let $H: G \mapsto \mathbb{R}$ and $l: V \mapsto \mathbb{R}$ be smooth maps. Let $v_{R}^{\phi}$ and $v_{L}^{\phi}$ be the spatial and convective velocities defined by (2) and (4), respectively. We define $\mathscr{F}_{R}$ on the set of paths in $G_{R}$ such that $\phi(0)=I d_{\Omega}$ by

$$
\mathscr{F}_{R}(\phi(t))=\int_{0}^{1} \ell\left(v_{R}^{\phi}(t)\right) \mathrm{d} t+H(\phi(1)) .
$$


Respectively, $\mathscr{F}_{L}(\phi)$ is defined on the set of paths in $G_{L}$ by

$$
\mathscr{F}_{L}(\phi(t))=\int_{0}^{1} \ell\left(v_{L}^{\phi}(t)\right) \mathrm{d} t+H(\phi(1)) .
$$

Then,

$$
\mathscr{F}_{R}(\phi(t))=\mathscr{F}_{L}\left(\phi(1) \phi^{-1}(1-t)\right),
$$

and as a consequence, the minimizers of $\mathscr{F}_{R}$ and $\mathscr{F}_{L}$ are in one to one bijection by the map $\phi(t) \mapsto \phi(1) \phi^{-1}(1-t)$.

Proof. Let $\psi: t \rightarrow \phi(1) \phi^{-1}(1-t)$. Changing the variable $t \mapsto 1-t$, and then applying the Lemma, we have

$$
\int_{0}^{1} \ell\left(v_{R}^{\phi}(t)\right) \mathrm{d} t=\int_{0}^{1} \ell\left(v_{R}^{\phi}(1-t)\right) \mathrm{d} t=\int_{0}^{1} \ell\left(v_{L}^{\psi}(t)\right) \mathrm{d} t .
$$

Since $\psi(0)=\phi(0)=I d_{\Omega}$ and $\psi(1)=\phi(1)$, the result follows.

Remark 5. 1. Generically, changing from right- to left- invariant Lagrangian does not change the endpoint of the optimal path.

2. The correspondence also holds for the boundary value problem, i.e. when $\phi(1)$ is fixed.

3. One can use a time-dependent Lagrangian $\ell(v, t)$ if $\ell(v, 1-t)=\ell(v, t)$ for all $t \in[0,1]$.

4. If the term $H$ is replaced by a path-dependent term, then the result does not hold any more.

A direct application of the previous proposition to the case of the kinetic energy defined by $\ell(v):=\frac{1}{2}\|v\|_{V}^{2}$ and $H(\phi)=E\left(\phi_{1} \cdot I, J\right)$ gives the following corollary. The existence of minimizers for these functionals is guaranteed by [36].

Corollary 1. [Equivalence of Optimal Matches in Left-and Right- LDM] Consider the problem of minimising

$$
\mathscr{J}(\phi)=\frac{1}{2} \int_{0}^{1}\|v(t)\|_{V}^{2} \mathrm{~d} t+E\left(\phi_{1} \cdot I, J\right)
$$

for $\phi_{0}=I d_{\Omega}$, and with either constraint

$$
\partial_{t} \phi_{t}=d \phi_{t} \cdot v_{t} \quad(\text { Left-LDM constraint })
$$

or

$$
\partial_{t} \phi_{t}=v_{t} \circ \phi_{t} \quad(\text { Right-LDM constraint }) .
$$

Then

1. The optimal endpoint $\phi_{1}$ is the same with either constraint.

2. If $\phi_{t}$ minimises $\mathscr{J}$ in Left-LDM, then $\psi_{t}:=\phi_{1-t}^{-1} \circ \phi_{1}$ minimises $\mathscr{J}$ in RightLDM. 
3. If $\psi_{t}$ minimises $\mathscr{J}$ in Right-LDM, then $\phi_{t}:=\psi_{1} \circ \psi_{1-t}^{-1}$ minimises $\mathscr{J}$ in Left$L D M$.

Optimal paths in Left-LDM are left-geodesics, while optimal paths in Right-LDM are right-geodesics.

In summary, the optimal diffeomorphism $\phi_{1}$ is the same in both approaches, but there are two optimal paths from $I d$ to $\phi_{1}$ : one left- and one right- geodesic. These two paths are illustrated in the following diagram.

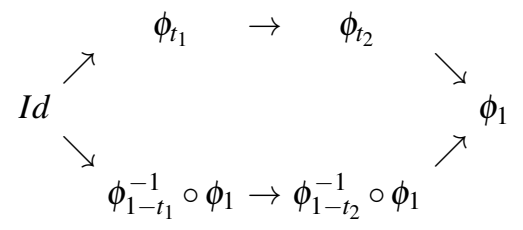

When left- (resp. right-) geodesics act on a image, the resulting paths in shape space are left- (resp. right-) geodesics. An example is given in Figure 1.
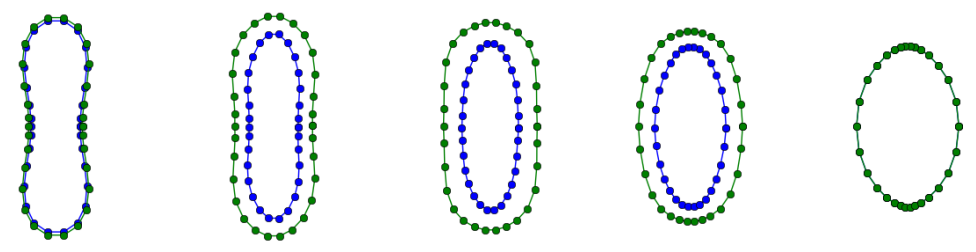

Fig. 1 This figure shows snapshots of two deformations from the left-most source image to the right-most target image. The green curves show the optimal Right-LDM path (a right-geodesic), while blue curves show the optimal Left-LDM path (a left-geodesic). Note that the paths are different, though both arrive at an exact match. The right-metric length of the green geodesic equals the left-metric length of the blue geodesic.

\section{Geodesic flow of left-invariant metrics}

We have considered minimisers of (1), which are geodesics. We now consider the corresponding initial value problem in which only $\phi(0)=I d$ is fixed. The minimisers $\phi(t)$ evolve according to Euler-Lagrange equations which are equivalent, in the Right-LDM case, to the EPDiff equation [19],

$$
\frac{d}{d t} \frac{\partial l}{\partial v}=-\operatorname{ad}_{v}^{*} \frac{\partial l}{\partial v}
$$


together with the spatial velocity constraint. This formulation leads to the momentum representation of diffeomorphisms, and further to the special pulson solutions, which correspond to image landmarks and have applications to optimization schemes [34, 7] and to the statistical description of images [23]. We now discuss the corresponding concepts in Left-LDM.

The first term of (1) with fixed endpoints may be expressed as $\int_{0}^{1} l(v(t)) d t$ where $l$ is the kinetic energy Lagrangian defined by

$$
l(v):=\frac{1}{2}\|v\|_{V}^{2}
$$

and $v(t)$ is the convective velocity of $\phi(t)$, defined in (4). The minima of this problem, with given endpoints $\phi(0)$ and $\phi(1)$, are left-geodesics, as defined in the previous section. There is a question of the well-posedness of the boundary value problem that defines these "left-geodesics". However, from the equivalence with Right-LDM shown in Section 3, it follows that the problem is well-posed for the same norms for which the corresponding problem in Left-LDM is well-posed. In addition, the Euler-Poincaré equation is available via this equivalence and let us point out that left-reduction is not needed here.

Euler-Poincaré equation. Using the equivalence with Right-LDM, under mild conditions on $H$ in (21), left-geodesics minimising (21) satisfy the left Euler-Poincaré equation [19],

$$
\frac{d}{d t} \frac{\partial l}{\partial v}=\mathrm{ad}_{v}^{*} \frac{\partial l}{\partial v}
$$

This equation can be expressed in terms of the convective momentum,

$$
p(t):=\frac{\partial l}{\partial v},
$$

as $\frac{d}{d t} p=\mathrm{ad}_{v}^{*} p$. In Euclidean coordinates, the Euler-Poincaré equation takes the following form, called EPDiff-left,

$$
\frac{\partial \mathbf{p}}{\partial t}=\operatorname{ad}_{\mathbf{v}}^{*} \mathbf{p}:=\mathbf{v} \cdot \nabla \mathbf{p}+(\nabla \mathbf{v})^{T} \cdot \mathbf{p}+\mathbf{p}(\operatorname{div} \mathbf{v}),
$$

where $(\nabla \mathbf{v})^{T} \cdot \mathbf{p}:=\sum_{j} p_{j} \nabla v^{j}$. If the norm is defined in terms of a kernel $K_{\sigma}$ as in (3), then $v=K_{\sigma} \star p$ and

$$
l=\frac{1}{2} \int_{0}^{1}\left\langle p(t), K_{\sigma} \star p(t)\right\rangle_{L^{2}} \mathrm{~d} t .
$$

Conservation law. Given the convective velocity constraint (4), the left-invariant Euler-Poincaré equation is equivalent to (see [19]) 


$$
0=\frac{d}{d t} \operatorname{Ad}_{\phi}^{*} \frac{\partial l}{\partial v}=\left(\phi^{-1}\right)^{*} p
$$

This is a conservation law, with the conserved quantity being spatial momentum,

$$
m(t):=\left(\phi^{-1}\right)^{*} p(t)=\left(\phi^{-1}\right)^{*} \frac{\partial l}{\partial v} .
$$

Note that this reverses the Right-LDM situation, where convective momentum is preserved and spatial momentum evolves according to EPDiff-right.

Pulsons. Singular "pulson" solutions may be found by making the following ansatz [13],

$$
\mathbf{p}(t)=\sum_{a=1}^{N} \mathbf{P}_{a}(t) \boldsymbol{\delta}\left(\mathbf{x}-\mathbf{Q}_{a}(t)\right) .
$$

It is known [17] that this momentum ansatz defines an equivariant momentum map

$$
J_{\text {Sing }}: T^{*} \operatorname{Emb}\left(S, R^{n}\right) \rightarrow \mathscr{X}\left(R^{n}\right)^{*}
$$

called the singular solution momentum map, where here $S$ is a finite set of $N$ points indexed by $a$. It is the momentum map for the cotangent-lift of the left action of $\operatorname{Diff}\left(R^{n}\right)$ on $\operatorname{Emb}\left(S, R^{n}\right)$. It follows from general theory (see e.g. [19]) that $J_{\text {Sing }}$ is a Poisson map with respect to the canonical symplectic form on $T^{*} E m b\left(S, R^{n}\right)$ and the right Lie-Poisson bracket on $\mathscr{X}\left(R^{n}\right)^{*}$. Thus the EPDiff-right equations pull back to canonical Hamiltonian equations in $Q$ and $P$, with respect to Hamiltonian

$$
H=\sum_{a, b=1}^{N}\left(\mathbf{P}^{a}(t) \cdot \mathbf{P}^{b}(t)\right) K\left(\mathbf{Q}^{a}(t), \mathbf{Q}^{b}(t)\right) .
$$

These are the singular pulson solutions discussed in [13] and elsewhere. It also follows, applying a time reversal, that the EPDiff-left equations (27) pull back to time-reversed canonical Hamiltonian equations in $Q$ and $P$, with respect to the same Hamiltonian:

$$
\begin{aligned}
\frac{\partial}{\partial t} \mathbf{Q}_{a}(t) & =-\sum_{b=1}^{N} \mathbf{P}_{b}(t) K\left(\mathbf{Q}_{a}(t), \mathbf{Q}_{b}(t)\right) \\
\frac{\partial}{\partial t} \mathbf{P}_{a}(t) & =\sum_{b=1}^{N}\left(\mathbf{P}_{a}(t) \cdot \mathbf{P}_{b}(t)\right) \frac{\partial}{\partial \mathbf{Q}_{a}} K\left(\mathbf{Q}_{a}(t), \mathbf{Q}_{b}(t)\right)
\end{aligned}
$$

These are the equations of the pulson solutions of EPDiff-left. Note that they are nearly the same equations as for the pulson solutions of EPDiff-right, with two important differences: (i) there is a time-reversal; and (ii) $Q_{a}(t)$ is not the spatial location of particle $a$ at time $t$, but instead it is an "anti-particle's location" in body coordinates, i.e. the location in body coordinates corresponding to a fixed spatial location $Q_{a}(0)$. This follows from the conservation of spatial momentum. Similar observations apply to higher-dimensional singular solutions (filaments, sheets, etc.). 
All of the results in this section can be either verified directly, making minor changes to the well-known proofs for right-geodesics (the flow of EPDiff-right), or deduced from the correspondence between left and right geodesics in Section 3.

\section{Spatially varying metrics and non-local symmetries}

Regarding applications, a crucial point consists in defining the metric which can be viewed as a parameter to be tuned accordingly with data. In the classical Right-LDM picture, due to translation and rotation symmetry, the class of metrics is rather small. In contrast, the Left-LDM model enables the use of many more types of kernels. In particular, kernels that incorporate non-local correlations. A striking example is the brain development where a symmetry at large scale between the left and the right parts of the brain can be exploited in order to improve the image matching quality. Of course, it is natural to ask for soft symmetry in practical applications rather than perfect symmetry. We give hereafter an explicit example of a kernel satisfying those requirements.

Let us first present the case of perfect symmetry: Let $\Pi: V \mapsto V$ be the symmetry of interest, which is a continuous linear operator on the space of vector fields $V$ that satisfies $\Pi^{2}=i d$. For instance, in $\mathbb{R}^{2}$ if $v=\left(v_{1}, v_{2}\right)$, the example showed in the simulation is $\left.\Pi\left(\left(v_{1}, v_{2}\right)\right)\right)=\left(u_{1}, u_{2}\right)$ where $u_{1}(x, y)=-v_{1}(-x, y)$ and $u_{2}(x, y)=$ $v_{2}(-x, y)$. The set of vector fields $v$ satisfying the symmetry condition $\Pi(v)=v$ is thus a closed linear subspace denoted by $V_{1}$, which may be endowed with the induced norm or alternatively with:

$$
\left\|v_{1}\right\|_{V_{1}}^{2}=\min _{v \in V}\left\{\|v\|_{V}^{2} \mid \frac{1}{2}(v+\Pi(v))=v_{1}\right\} .
$$

In general, those two norms do not coincide, unless $\Pi$ is self-adjoint which is the case in our example. We prefer the metric (32) since the kernel associated with that metric is given by:

$$
K_{V_{1}}=\frac{1}{4}(I d+\Pi) \circ K \circ\left(I d+\Pi^{*}\right) .
$$

Since, in our example, $\Pi$ is self-adjoint, we can simplify the expression of $K_{V_{1}}$ to get

$$
K_{V_{1}}=\frac{1}{2}(I d+\Pi) \circ K .
$$

The above kernel $K_{V_{1}}$ will produce perfect symmetry which is not desired as mentioned above. However, we can modify it to allow for a variable degree of symmetry. For example, consider the class of kernels

$$
K=(I d+c \Pi) \circ K_{\sigma},
$$


where the strength of the symmetry ranges from none at $c=0$ to perfect symmetry at $c=1$. It is also natural to introduce a mixture of kernels with different length scales, to account for local discrepancies in the deformation field, i.e. which means using

$$
K=(I d+c \Pi) \circ K_{\sigma_{1}}+K_{\sigma_{2}},
$$

where $\sigma_{1}, \sigma_{2}$ are the scale parameters of the kernels, for example the standard deviation of the Gaussian kernel. In particular, it is natural to use $\sigma_{1}>\sigma_{2}$ to account for large scale symmetry. Looking at the form of the kernel (36), it is tempting to introduce a spatially varying coefficient that accounts for more or less symmetry or importance of a given kernel. Therefore, the final example of spatially-varying kernel is the following: Let $K_{i}$ be $n$ kernels and $\chi_{i}: \Omega \mapsto[0,1]$ be $n$ smooth functions such that $\sum_{i=1}^{n} \chi_{i}=1$ then we consider

$$
K=\sum_{i=1}^{n} \chi_{i} K_{i} \chi_{i}
$$

This kernel is associated to the following variational interpretation:

$$
\|v\|^{2}=\min _{\left(v_{1}, \ldots, v_{n}\right) \in V_{1} \times \ldots \times V_{n}}\left\{\sum_{i=1}^{n}\left\|v_{i}\right\|_{V_{i}}^{2} \mid \sum_{i=1}^{n} \chi_{i} v_{i}=v\right\} .
$$

We note that Formula (38) is a simple generalization of mixtures of kernels, which are explained in detail in [6].

\section{Experiments}

In the following experiments, we are interested in deformations generated by the Left-LDM model using spatially dependent kernels that incorporate the soft symmetry constraint proposed in Section 5. By the equivalence proven in Section 3, the final deformation is also given by the corresponding Right-LDM model, so all of the numerical results presented below have been computed using the standard gradient descent optimization method for the Right-LDM model detailed in [6].

We registered two images out of the LPBA40 dataset [28]. We considered Subjects 8 and 9 of the dataset. The images were resampled to a resolution of $1 \mathrm{~mm}$ and rigidly aligned. We then extracted corresponding 2D slices from the two aligned images. Finally, we simulated a large lesion in the slice from Subject 8. A mask was also constructed, by dilating the original lesion location mask 8 times, each time using a $3 \times 3$ structuring element. This mask was used to omit lesioned areas from calculation of the image dissimilarity term, and also to mask the updated momenta before smoothing. Registered images are shown in Fig. 2.

We registered the lesioned images with LDM as described above, using two kinds of kernel: a standard translationally-invariant sum of Gaussian kernels (non- 

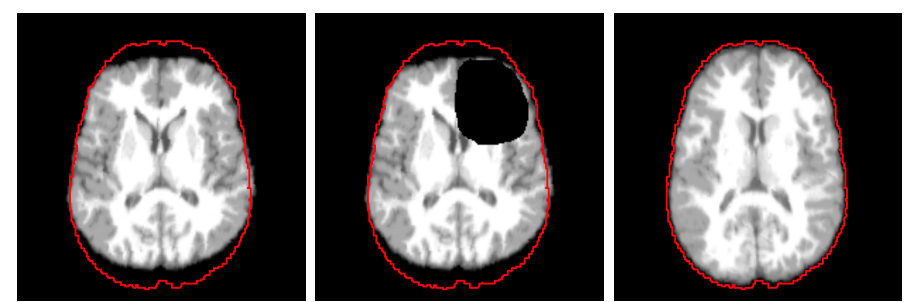

Fig. 2 (From left to right) 2D slice from Subject 8 of the LPBA40 dataset; same slice with a simulated lesion (source image); and corresponding 2D slice from Subject 9 (target image). The red isoline represents the surface of Subject 9's brain.
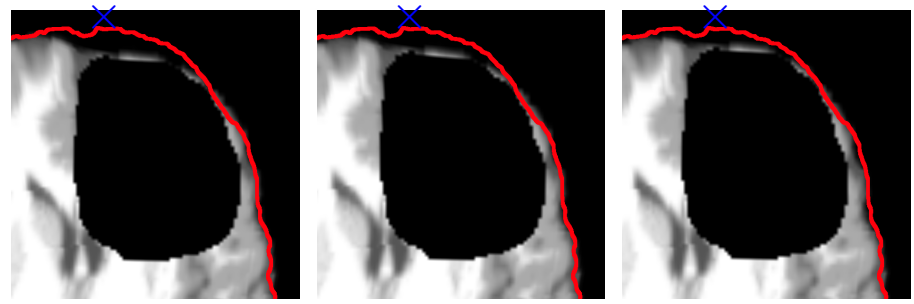

Fig. 3 Source images around the simulated lesion deformed using the registration strategies 1 and 2 of section 6. From left to right, the registration strategies were: non-symmetric kernel (strategy 1 ); symmetric kernel (strategy 2) with symmetry weighting factor $c=0.1$; and symmetric kernel (strategy 2) with symmetry weighting factor $c=1$ (pure symmetry at large scale). The red isoline (surface of the target) and the blue cross are always at the same location, to visualize the influence of the symmetric kernel.

symmetric); and a spatially-varying kernel that softly enforces a left-right symmetry:

1. (non-symmetric) the sum of two Gaussian kernels $K_{\sigma_{1}}+K_{\sigma_{2}}$, where $\sigma_{1}=25 \mathrm{~mm}$ and $\sigma_{2}=7 \mathrm{~mm}$, as in $[4,27]$.

2. (symmetric) the sum of a large-scale symmetrised kernel with a small-scale Gaussian kernel, $K_{\sigma_{1}}+c \Pi K_{\sigma_{1}}+K_{\sigma_{2}}$, where $\Pi$ is a reflection about the vertical line dividing the two hemispheres. The values of $\sigma_{1}$ and $\sigma_{2}$ are the same as above, and $c$ takes values 0.1 (weak symmetry), 0.5 or 1.0 (pure symmetry at large scale).

For comparison, we have also performed LDM registration of the unlesioned images using kernel (1) without a mask.

Deformed images are shown in Fig. 3 and deformation magnitudes in the $x$ direction (horizontal) are shown in Fig. 4. We can see in Fig. 3 that modeling a symmetry in the left and right sides of the brain allows partial compensation for the information missing in the lesion. The deformations estimated in the lesion are indeed almost only due to the symmetry as clearly emphasized in Fig. 4. It is also interesting to remark that the most similar deformation to the one obtained without the 
lesion (image (a) in Fig. 4) is not the one obtained using pure symmetry on the large scale (image (e) in Fig. 4), but the one obtained using a factor 0.5 on the symmetry (image (d) in Fig. 4). In this case, the symmetry plausibly compensates for the missing information at a large scale in the lesion but does not penalize too much the estimation of the deformations in the region symmetric to the lesion.
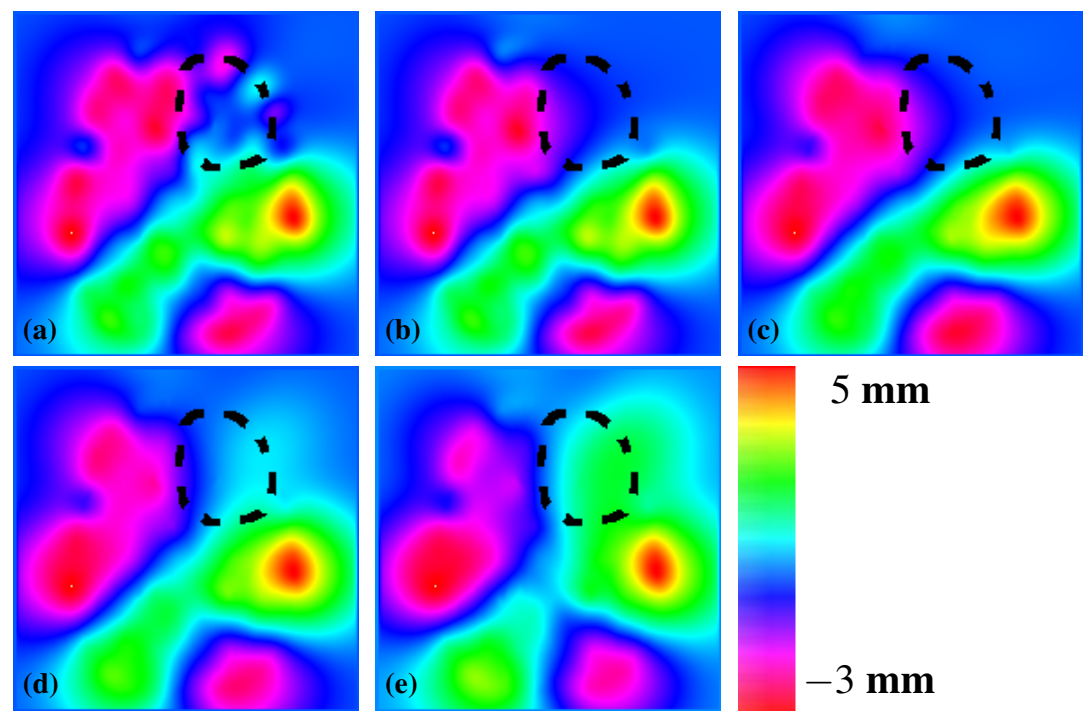

Fig. 4 Deformation magnitude in the $x$ direction (horizontal) estimated using the different registration strategies in Section 6. Results were obtained by registering the images without (a) and with (b-e) the lesion. A mixture of Gaussian kernels was used in (a-b). In (c),(d),(e) a similar mixture of kernels was used, but with a symmetry at the large scale weighted by the factors $0.1,0.5$ and 1 , respectively. The dashed curve represents the boundary of the simulated lesion.

\section{Discussion}

We have introduced a new perspective on diffeomorphic image matching, based on left- (rather than right-) invariant metrics. For inexact matching with Left-LDM, the optimal diffeomorphism $\phi(1)$ is the same as for Right-LDM (i.e., the usual LDDMM), however there are two different optimal paths from the identity to $\phi(1)$ in the diffeomorphism group: one left- and one right- geodesic. This difference could become significant if a time-dependent similarity measure were used.

In the Left-LDM setting, it is clear that spatially-varying and nonisotropic kernels describe variable deformability properties of the source image. We have shown, in a numerical experiment, the value of spatially-varying kernels as problem-specific 
regularisation terms in inexact matching. In particular, in a model of a lesioned brain image, we found that a kernel including a large-scale soft symmetry constraint was successful in compensating for missing information in the lesion area.

Through the relationship between Left- and Right- LDM, it also becomes apparent that spatially-varying and directionally-dependent kernels in Right-LDM have an interpretation in terms of local deformability properties of the source image, which has not been remarked upon in the literature.

One very promising avenue for further work is to replace ad-hoc regularisation choices with automatically learnt ones, as has been done by Simpson et al. [30] for global regularisation parameters. Similar methods could be developed for spatially-varying and directionally-dependent regularisation, based on a generative Left-LDM model. Given a template image $I$, the LDM functional (1) can be interpreted as a $\log$ probability density function on pairs of initial vector fields $v(0)$ and images $J$ :

$$
\begin{aligned}
\log P(v(0), J \mid I, \lambda, \sigma) & =\log P(v(0) \mid \sigma)+\log P(J \mid v(0), I, \lambda) \\
& =\frac{1}{2} \int_{0}^{1}\|v(t)\|_{V_{\sigma}}^{2} d t+\frac{\lambda}{2}\|\phi(1) \cdot I-J\|_{L^{2}}^{2},
\end{aligned}
$$

with the constraint (4) determining $v(t)$ and $\phi$ from $v(0)$. This could in theory be marginalised over $v$ to get $P(J)$. Both the regularisation parameters $\sigma$ and the noise parameters $\lambda$ could be spatially-varying, possibly expressed in terms of labels associated with the template. A variety of more or less standard methods could be used to optimise the parameters for a population of targets, including Bayesian methods related to those in $[1,8]$.

\section{References}

1. Allassonnière, S., Amit, Y., Trouvé, A.: Towards a coherent statistical framework for dense deformable template estimation. J. R. Statist. Soc. B 69(1), 3-29 (2007)

2. Arsigny, V., Commowick, O., Pennec, X., Ayache, N.: A Log-Euclidean framework for statistics on diffeomorphisms. In: R. Larsen, M. Nielsen, J. Sporring (eds.) Proc. MICCAI06, vol. 4190, pp. 924-931. Springer-Verlag (2006)

3. Ashburner, J.: A fast diffeomorphic image registration algorithm. NeuroImage 38, 95-113 (2007)

4. Beg, M.F., Miller, M.I., Trouvé, A., Younes, L.: Computing large deformation metric mappings via geodesic flows of diffeomorphisms. Int. J. Comput. Vision 61(2), 139-157 (2005)

5. Bruveris, M., Gay-Balmaz, F., Holm, D., Ratiu, T.: The momentum map representation of images. Journal of Nonlinear Science 21(1), 115-150 (2011)

6. Bruveris, M., Risser, L., Vialard, F.: Mixture of kernels and iterated semidirect product of diffeomorphisms groups. Multiscale Modeling and Simulation 10(4), 1344-1368 (2012)

7. Cotter, C.J.: The variational particle-mesh method for matching curves. Journal of Physics A: Mathematical and Theoretical 41(34), 344,003 (2008)

8. Cotter, C.J., Cotter, S.L., Vialard, F.X.: Bayesian data assimilation in shape registration. Inverse Problems 29(4), 045,011 (2013)

9. Cotter, C.J., Holm, D.D.: Continuous and discrete Clebsch variational principles. Foundations of Computational Mathematics 9, 221-242 (2009) 
10. Dupuis, P., Grenander, U., Miller, M.I.: Variational problems on flows of diffeomorphisms for image matching. Quart. Appl. Math. 56, 587-600 (1998)

11. Ebin, D.G., Marsden, J.E.: Groups of diffeomorphisms and the motion of an incompressible fluid. Ann. of Math 92, 102-163 (1970)

12. Fischer, A.E., Marsden, J.E.: The Einstein evolution equations as a first-order quasi-linear symmetric system, I. Communications in Mathematical Physics 28, 1-38 (1972)

13. Fringer, O., Holm, D.: Integrable vs nonintegrable geodesic soliton behavior. Physica D 150, 237-263 (2001)

14. Gay-Balmaz, F., Holm, D.D., Ratiu, T.S.: Geometric dynamics of optimization. Comm. in Math. Sciences 11(1), 163-231 (2013)

15. Gay-Balmaz, F., Marsden, J.E., Ratiu, T.S.: Reduced variational formulations in free boundary continuum mechanics. J Nonlinear Sci 22, 463-497 (2012)

16. Gay-Balmaz, F., Ratiu, T.S.: Clebsch optimal control formulation in mechanics. Journal of Geometric Mechanics 3, 47-79 (2011)

17. Holm, D.D., Marsden, J.E.: Momentum maps and measure-valued solutions (peakons, filaments and sheets) for the EPDiff equation. Progr. in Math. 232, 203-235 (2004)

18. Holm, D.D., Marsden, J.E., Ratiu, T.: The Hamiltonian structure of continuum mechanics in material, inverse material, spatial, and convective representations. In: Hamiltonian Structure and Lyapunov Stability for Ideal Continuum Dynamics, vol. 100, pp. 11-124. Presses Univ. Montréal, Montréal (1986)

19. Holm, D.D., Schmah, T., Stoica, C.: Geometric Mechanics and Symmetry: From Finite to Infinite Dimensions. Oxford: Clarendon Press (2009)

20. Holm, D.D., Tilak Ratnanather, J., Trouvé, A., Younes, L.: Soliton Dynamics in Computational Anatomy. eprint arXiv:nlin/0411014 (2004)

21. Holm, D.D., Trouvé, A., Younes, L.: The Euler Poincaré theory of metamorphosis. Quart. Appl. Math (2008)

22. Kudryavtsev, L.: Implicit function. Encyclopedia of Mathematics (2011). URL http://www.encyclopediaofmath.org

23. Miller, M., Qiu, A.: The emerging discipline of computational functional anatomy. NeuroImage 45, S16-S39 (2009)

24. Omori, H.: Infinite dimensional Lie tranformation groups, Lecture Notes in Mathematics, vol. 427. Springer-Verlag, Berlin (1974)

25. Risser, L., Vialard, F.X., Baluwala, H.Y., Schnabel, J.A.: Piecewise-diffeomorphic image registration: Application to the motion estimation between 3D CT lung images with sliding conditions. Medical Image Analysis 17, 182-193 (2013)

26. Risser, L., Vialard, F.X., Wolz, R., Holm, D.D., Rueckert, D.: Simultaneous fine and coarse diffeomorphic registration: Application to the atrophy measurement in alzheimer's disease. In: MICCAI 2010, Lecture Notes in Computer Science, vol. 6362, pp. 610-617. Springer, Berlin (2010)

27. Risser, L., Vialard, F.X., Wolz, R., Murgasova, M., Holm, D.D., Rueckert, D.: Simultaneous Multi-scale Registration Using Large Deformation Diffeomorphic Metric Mapping. IEEE Transactions on Medical Imaging 30(10), 1746-1759 (2011)

28. Shattuck, D.W., Mirza, M., Adisetiyo, V., Hojatkashani, C., Salamon, G., Narr, K.L., Poldrack, R.A., Bilder, R.M., Toga, A.W.: Construction of a 3D probabilistic atlas of human cortical structures. NeuroImage 39, 1064-80 (2008)

29. Simo, J.C., Marsden, J.E., Krishnaprasad, P.S.: The Hamiltonian structure of nonlinear elasticity: the material, spatial and convective representations of solids, rods and plates. Arch. Ration. Mech. Anal. 104, 125-183 (1988)

30. Simpson, I., Schnabel, J., Groves, A., Andersson, J., Woolrich, M.: Probabilistic inference of regularisation in non-rigid registration. NeuroImage 59(3), 2438-51 (2012)

31. Trouvé, A.: Diffeomorphic groups and pattern matching in image analysis. Int. J. Comput. Vision 28, 213-221 (1998)

32. Trouvé, A., Younes, L.: Metamorphoses through lie group action. Foundations of Computational Mathematics 5, 173-198 (2005) 
33. Vercauteren, T., Pennec, X., Perchant, A., Ayache, N.: Diffeomorphic demons: efficient nonparametric image registration. Neuroimage 45(1 Suppl), S61-S72 (2009)

34. Vialard, F.X., Risser, L., Rueckert, D., Cotter, C.J.: Diffeomorphic 3D image registration via geodesic shooting using an efficient adjoint calculation. International Journal of Computer Vision 97(2), 229-241 (2012)

35. Yosida, K.: Functional Analysis, Die Grundlehren der mathematischen Wissenschaften in Einzeldarstellungen, vol. 123. Springer-Verlag, Berlin, New York (1965)

36. Younes, L.: Shapes and Diffeomorphisms. Springer (2010) 